\section{A Real World Cost Comparison between Chinese Patients with Coronary Heart Disease or Angina Pectoris using Salvianolate and without using Salvianolate: A Retrospective Database Analysis}

\section{Abstract}

Objective: To assess and compare 1-year cardiovascular events occurrence frequency and treatment cost among Chinese coronary heart disease (CHD) or angina pectoris patients who used salvianolate injection or non-user.

Method: Data were obtained from electronic medical record (EMR) database between 2012.01.01 2017.12.31 covering medical encounter records from two tier-3 general hospitals from Shanghai and Guangzhou in China. Patients were followed 12 months after drug initiation. T-test, Chi-square test, negative binomial regression and generalized linear model adjusting for demographics, and baseline characteristics including cardiovascular event (myocardial infarction, angina, transient ischemic attack, stroke, etcs) rate, inpatient length of stay, and healthcare resource consumption were used to test the difference between two groups.

Result: A total of 439 and 1848 patients were identified for salvianolate injection and control group, with mean (SD) age $75.42(12.09)$ and $69.15(12.79)(p<0.01)$ and male $43.28 \%$ and $52.38 \%$ ( $p<0.01$ ), respectively. During baseline, the mean frequency of cardiovascular events were 2.05 and 5.82 times for treatment and control group respectively $(p<0.01)$; the treatment cost for cardiovascular event were 4720.04 Chinese Yuan (CNY) and 3700.23 CNY for control group respectively $(p=0.11)$. During the follow-up period, the average number of cardiovascular events were 0.26 and 5.87 times for treatment and control group respectively $(p<0.01)$. Compared with non-user, mean per-year cost for salvianolate injection user was $29.58 \%$ lower (4069.21 vs. 5778.17 Chinese Yuan (CNY).

Conclusion: Among Chinese coronary heart disease and angina pectoris patients, those who used salvianolate injection had lower per-year cardiovascular event rate and spent less on cardiovascular events treatment.

Keywords: Cost; coronary heart disease (CHD); angina pectoris; salvianolate injection; China

\section{Yingrui Xu', Jianwei Xuan ${ }^{1 *}$, Hongying Liư ${ }^{2}$, Yue $\mathrm{GaO}^{3}$ and Linguo $\mathrm{Li}^{3}$}

1 Health Economic Research Institute, Sun Yat-Sen University, Guangzhou, China

2 GreenValley Inc, Shanghai, China

3 Shanghai Centennial Scientific Co. Ltd, Shanghai, China

\section{*Corresponding author: Jianwei Xuan \\ Exuanjw3@sysu.edu.cn}

Director and Professor, Health Economic Research Institute, Sun Yat-Sen University, Guangzhou, P. R. China.

Tel: +8639943405

Citation: Xu Y, Xuan J, Liu H, Gao Y, Li L (2018) A Real World Cost Comparison between Chinese Patients with Coronary Heart Disease or Angina Pectoris using Salvianolate and without using Salvianolate: A Retrospective Database Analysis. J Health Med Econ Vol.5 No.1:6

Received: August 28, 2018; Accepted: September 20, 2018; Published: October 01, 2018

\section{Introduction}

Cardiovascular disease (CVD) is the leading global cause of death, an estimated 17.7 million people died from CVDs in 2015 , representing $31 \%$ of all global deaths [1]. The number that is expected to grow to more than 23.6 million by 2030 [2]. According to China cardiovascular disease report 2016, the incidence of cardiovascular disease is still increasing recently with the population aging and life style change. Cardiovascular disease remains to be the No.1 cause of death in China, accounting for $40 \%$ all cause of death. The current population of cardiovascular disease patient is 290 million, among which the patient number of high blood pressure, stroke and coronary heart disease is 270 million, 13 million and 11 million respectively [3]. Coronary heart disease is within the group of cardiovascular diseases of which it is the most common type. In 2015 , the mortality rate 
of coronary heart disease in urban and rural areas in China was $110.67 / 100,000$ and $110.91 / 100,000$ respectively [4]. Coronary heart diseases are associated with substantial medical costs and social burden in China, which exerts a substantial economic impact on its national health system and society.

Coronary heart disease (CHD) is also called coronary artery disease; the underlying mechanism involves reduction of blood flow and oxygen to the heart muscle due to atherosclerosis of the arteries of the heart. It refers to a group of diseases which includes stable angina, unstable angina, myocardial infarction, and sudden cardiac death [5]. Risk factors include high blood pressure, smoking, diabetes, lack of exercise, obesity, high blood cholesterol, poor diet, depression, and excessive alcohol [6-8]. Secondary prevention of coronary heart disease (CHD) is for patients with existing CHD to strictly control risk factors and prevent recurrence of cardiovascular events and heart failure. The aim is to reduce the mortality and disability rate of coronary heart disease and improve overall survival and quality of life. Secondary prevention measures for coronary heart disease include nondrug interventions (therapeutic life style changes, exercise and rehabilitation) combined with drug therapy and comprehensive prevention and control of cardiovascular risk factors, which can help to maximize the prognosis of CHD patients [9].

Salvianolate injection is a patent traditional Chinese medicine (TCM) widely used for treating CHD in China. Salvianolate is derived from the dried root of salviae miltiorrhizae (Danshen) by means of water extraction. It is manufactured by Green valley Pharmacia (Shanghai, China). The major active component, magnesium lithospermate B (content $>85 \%$ ), is detected for quality control during production. The other components of salvianolate consist of different polyphenolate compounds. [10]. Danshen watersoluble substances have a very strong antioxidant activity due to a number of phenolic hydroxyl components [11]. Studies show that salvianolic acids $B$ inhibits the production of superoxide anion, prevent the formation of erythrocyte hemolysis induced by hydrogen peroxide and malondialdehyde (MDA), protects against glucose-induced intracellular oxidative damage, inhibits the activity of aldose reductase in vascular smooth muscle cell induced by high dose of glucose and endometrial hyperplasia [11-13].

Pharmacological studies indicated that salvianolate injection could protect cardiomyocytes via reducing the levels of proinflammatory cytokines and inhibiting reactive oxygen species production, and protect myocardiocytes against ischemia [14-16]. Clinical study showed that salvianolate injection in a dosage of $200 \mathrm{mg}(3.3 \mathrm{mg} / \mathrm{kg} /$ day for an adult weighing $60 \mathrm{~kg}$ ) is effective and safe for the treatment of $\mathrm{CHD}$ angina pectoris [17]. Systematic reviews indicated that the combined use of salvianolate injection and western medicine could improve the total effective rate and the electrocardiogram effective rate, and the safety of salvianolate injection was confirmed $[18,19]$.

The purpose of this study was to retrospectively assess and compare of the one-year cardiovascular event rate and total treatment cost among Chinese coronary heart disease (CHD) or angina pectoris patients who used salvianolate injection or not using the electronic medical record database from real world. The study result is expected to provide references for health professional workers and health policymakers to make clinical or health policy decisions about coronary heart disease treatment.

\section{Methods}

\section{Data source}

Data were obtained from electronic medical record (EMR) database between 2012.01.01 2017.12.31 covering medical encounter records from two tier-3 general hospitals (Huashan hospital in Shanghai and First affiliated hospital of Sun Yat-sen University in Guangzhou) in China. A total of 23,265 patients were diagnosed with coronary heart disease or angina pectoris. Of these, 2,287 patients (439 patients using salvianolate injection and 1,848 patients not using salvianolate injection at the index date) were included in the analysis. Patient flow is detailed in Figure 1.

A retrospective analysis was conducted using anonymised records between 1 January 2012 and 31 December 2017 from the two tier-3 general hospitals (Huashan Hospital and First affiliated hospital of sun yat-sen University).

Patients who diagnosed with coronary heart disease or angina pectoris at the index date; and had trackable data longer than 12 months of continuous follow-up after salvianolate injection initiation were included in this study. The time for first prescription of salvianolate injection during study time frame constituted index date. Because the data were retrospectively extracted and only anonymised records were used in the analysis, ethical approval and written informed patient consent were not required for this study.

Endpoint are defined and calculated as follow: per-year number of cardiovascular events (myocardial infarction, angina, transient ischemic attack, and stroke) and total treatment cost per-year including both inpatient and outpatient during the 12-months follow-up period.

Health care resource use is defined as inpatient and outpatient visits either for cardiovascular events or for all-cause disease during the patient follow-up period.

\section{Statistical analysis}

The patients' baseline characteristics were compared between the salvianolate injection and control group by 2-sample t-test for continuous variables and chi-square test for categorical variables and binomial regression and generalized linear model adjusting for demographics, baseline characteristics including cardiovascular events, inpatient days, and healthcare resource consumption.

A 2-sided significance level of 0.05 was used. Adjustments for multiplicity were not made due to the exploratory purpose. All analyses were conducted by R-3.4.4. 


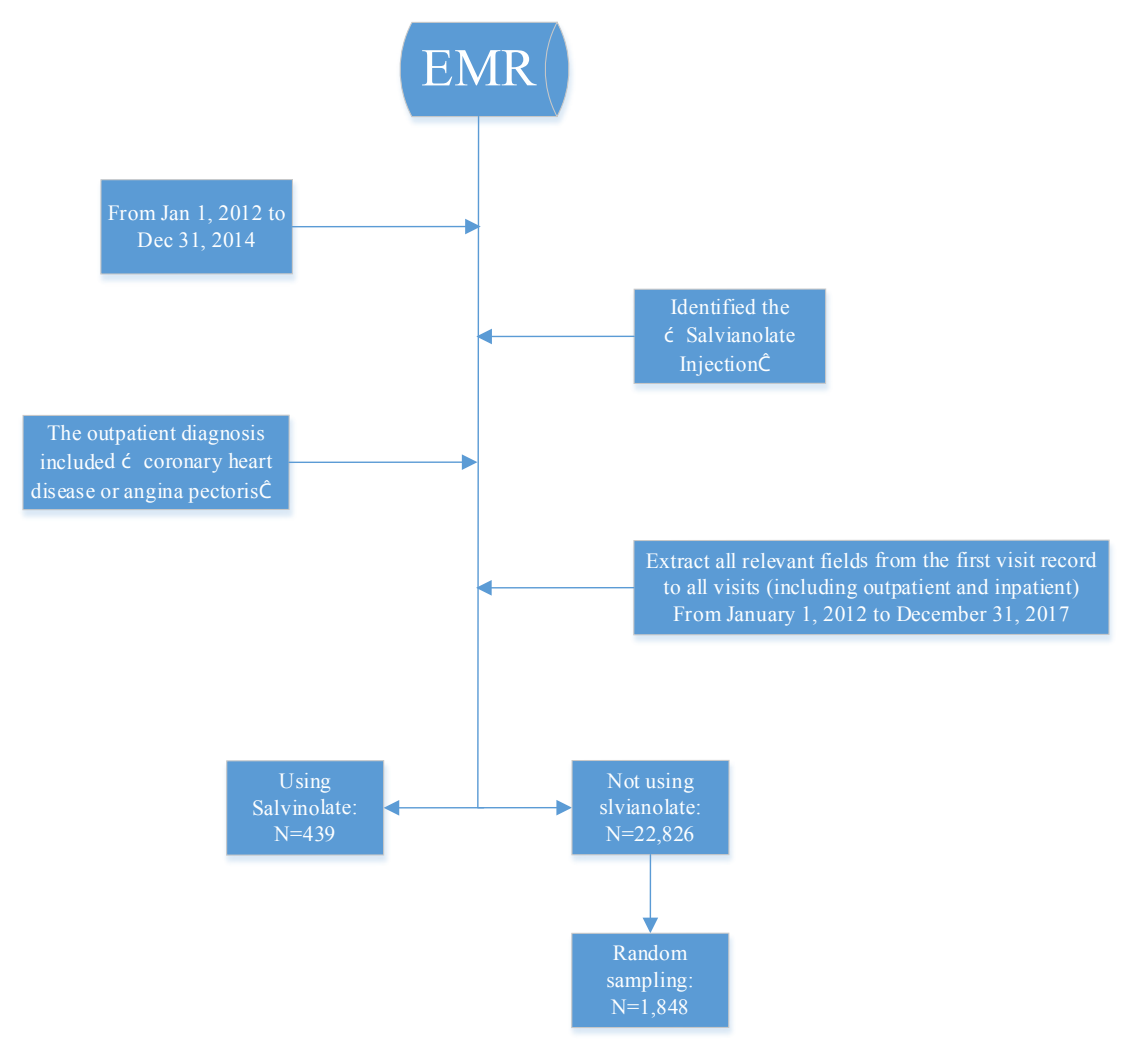

Abbreviations: EMR: Electronic Medical Record.

Figure 1 Patient flow chart.

\section{Results}

\section{Analysis population}

Between 1 January 2012 and 31 December 2017, a total of 439 patients of using salvianolate injection were diagnosed with coronary heart disease or angina pectoris, 22,826 patients of not using salvianolate injection were diagnosed with coronary heart disease or angina pectoris.

\section{Demographics and baseline clinical characteristics}

There were several statistically significant differences in the baseline characteristics between the using salvianolate and not using salvianolate groups (Table 1). Specifically, patients in the using salvianolate group had more inpatient visits for either major adverse cardiovascular events (MACE) or all cause, and spent less on medical costs for outpatient visits for either MACE or all cause (all $\mathrm{P}<0.05$ ).

\section{Salvianolate injection and no salvianolate injection after index date}

During the 12-month follow up period, no significant difference in total inpatient medical cost for all cause and MACE between two groups: the total inpatient medical cost for all cause unadjusted was 1,563.44 CNY and 1,258.41 CNY $(P=0.44)$, and the total inpatient medical cost for all cause adjusted was $2,858.76 \mathrm{CNY}$ and 1,468.25 CNY $(P=0.593)$, the total inpatient medical cost for
MACE unadjusted was 854.96 CNY and 596.79 CNY $(P=0.27)$, and the total inpatient medical cost for MACE adjusted was 831.93 CNY and 610.83 CNY ( $P=0.842)$ for the using salvianolate injection group and not, respectively.

\section{Health resource use for all cause}

There were several statistically significant differences between the two groups: the total medical cost for all cause unadjusted was 2,134.39 CNY and 3,542.67 CNY $(\mathrm{P}<0.001)$, the total medical cost for all cause adjusted was 4,069.21 CNY and 5,778.17 CNY $(P<0.001)$, total medical cost for all cause in outpatient unadjusted was 570.95 CNY and 2,284.26 CNY $(P<0.001)$, total medical cost for all cause in outpatient adjusted was 74.20 CNY and 7,400.09 CNY $(P<0.001)$, all-cause outpatient visit times unadjusted was 1.56 and $8.94(P<0.001)$, and all-cause outpatient visit times adjusted was 0.15 and $6.11(P<0.001)$ for using salvianolate and not, respectively (Figure 2 ).

\section{MACE-related health care resource use and total medical costs}

The total medical cost for MACE unadjusted was 1,084.86 CNY and 1,946.31 CNY $(P<0.001)$, and the total medical cost for MACE adjusted was 868.35 CNY and 2,034.93 CNY $(P<0.001)$; total medical cost for MACE in outpatient unadjusted was 229.89 CNY and 1,349.53 CNY $(P<0.001)$ and total medical cost for MACE in outpatient adjusted was 87.64 CNY and 1,355.22 CNY $(P<0.001)$, total medical cost for MACE in inpatient unadjusted was 854.96 
Table 1 Demographics and baseline characteristics.

\begin{tabular}{|c|c|c|c|}
\hline & \multirow[t]{2}{*}{ Using salvianolate ( $n=439$ ) } & Not using salvianolate & \multirow[t]{2}{*}{ P Value } \\
\hline & & $(n=1848)$ & \\
\hline Age, mean (SD), y & 75.42 (12.09) & $69.15(12.78)$ & $<0.0001$ \\
\hline$\geq 60$, No. (\%) & $38.00(8.66)$ & $368.00(19.91)$ & $<0.0001$ \\
\hline Female, No. (\%) & $249.00(56.72)$ & $880.00(47.62)$ & 0.0007 \\
\hline \multicolumn{4}{|c|}{ Reimbursement Type, No. (\%) } \\
\hline 0 & $345.00(78.59)$ & $1,247.00(67.48)$ & \multirow[t]{3}{*}{$<0.0001$} \\
\hline 1 & $48.00(10.93)$ & $55.00(2.98)$ & \\
\hline 2 & $46.00(10.48)$ & $546.00(29.55)$ & \\
\hline \multicolumn{4}{|c|}{ Inpatient visits for all cause, No. (\%) } \\
\hline 0 & $360.00(82.00)$ & $1,695.00(91.72)$ & \multirow[t]{2}{*}{$<0.0001$} \\
\hline$>1$ & $79.00(18.00)$ & $153.00(8.28)$ & \\
\hline Outpatient visits for all cause, & \multirow[t]{2}{*}{$4.76(14.64)$} & \multirow[t]{2}{*}{$12.46(16.40)$} & \multirow[t]{2}{*}{$<0.0001$} \\
\hline mean (SD), No. & & & \\
\hline Outpatient visits for MACE, & \multirow[t]{2}{*}{$1.87(3.02)$} & \multirow[t]{2}{*}{$5.75(7.32)$} & \multirow[t]{2}{*}{$<0.0001$} \\
\hline mean (SD), No. & & & \\
\hline Total medical costs for all cause, & \multirow[t]{2}{*}{$8,105.39(16,160.00)$} & \multirow[t]{2}{*}{$4,770.96(9,569.62)$} & \multirow[t]{2}{*}{$<0.0001$} \\
\hline mean (SD), CNY & & & \\
\hline Total medical costs for MACE, & \multirow[t]{2}{*}{$4,720.04(12,520.00)$} & \multirow[t]{2}{*}{$3,700.23(8,962.15)$} & \multirow[t]{2}{*}{0.1077} \\
\hline mean (SD), CNY & & & \\
\hline Total inpatient medical costs for all cause, mean (SD), CNY & $6,180.86(15,880.00)$ & $1,926.88(8,798.70)$ & $<0.0001$ \\
\hline Total inpatient medical costs for MACE, mean (SD), CNY & $3,395.27(12,290.00)$ & $1,381.14(7,619.98)$ & 0.0011 \\
\hline Total outpatient medical costs for all cause, mean (SD), CNY & $1,924.53(3,108.00)$ & $2,879.72(3,514.31)$ & $<0.0001$ \\
\hline Total outpatient medical costs for MACE, mean (SD), CNY & $1,324.77(1,864.00)$ & $2,319.09(3,199.82)$ & $<0.0001$ \\
\hline
\end{tabular}

Abbreviations CNY: Renminbi, the official currency of China; SE: Standard Error of Mean; MACE: Major Adverse Cardiovascular Event; SD: Standard Deviation of Mean, No.: Number.

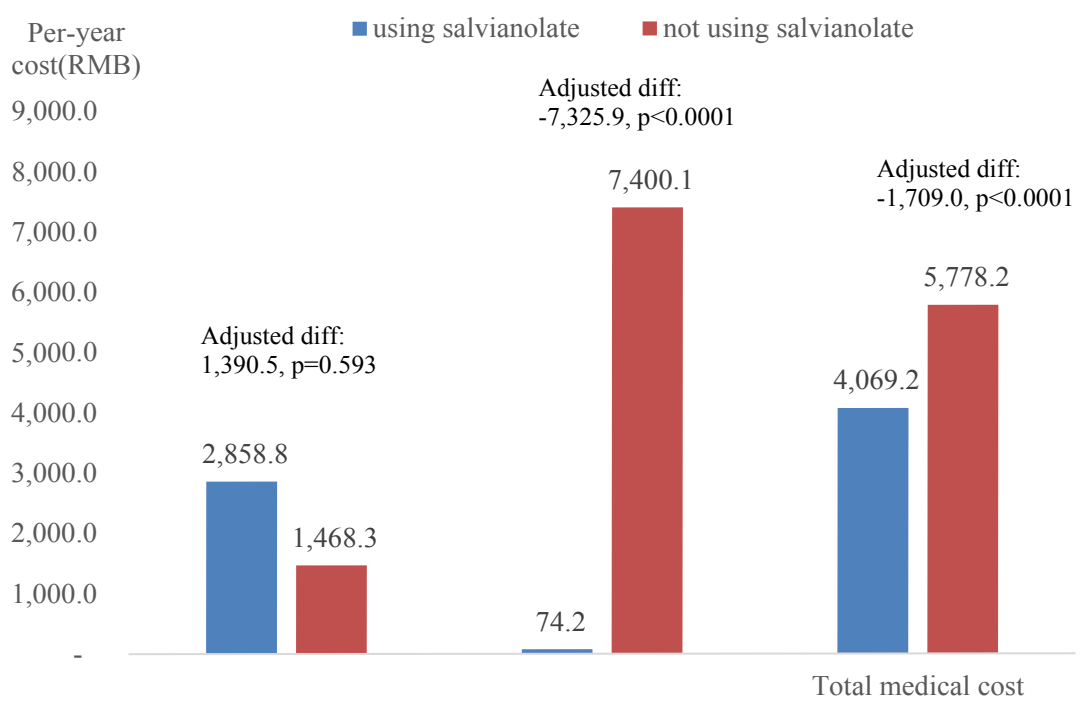

Abbreviations Diff, Difference; CNY: Renminbi, the Official Currency of China.

Figure 2 Total medical cost during 12 months follow up.

CNY and 596.79 CNY ( $P=0.265)$ and total medical cost for MACE in inpatient adjusted was 831.93 CNY and 610.83 CNY $(P=0.842)$ for using salvianolate and not, respectively (Figure 3 ).

\section{Cardiovascular related events during the follow-up}

Patients in using salvianolate group had less number of MACE
(0.26 vs. $5.87 ; P<0.0001$ [adjusted]), less inpatient days of MACE ( 0.00 vs. $0.36 ; P<0.001$ [adjusted]) and less number of outpatient MACE (0.15 vs. $6.11 ; \mathrm{P}<0.0001$ [adjusted]) compared with the not using salvianolate group during the 12-months follow-up period (Figure 4). 


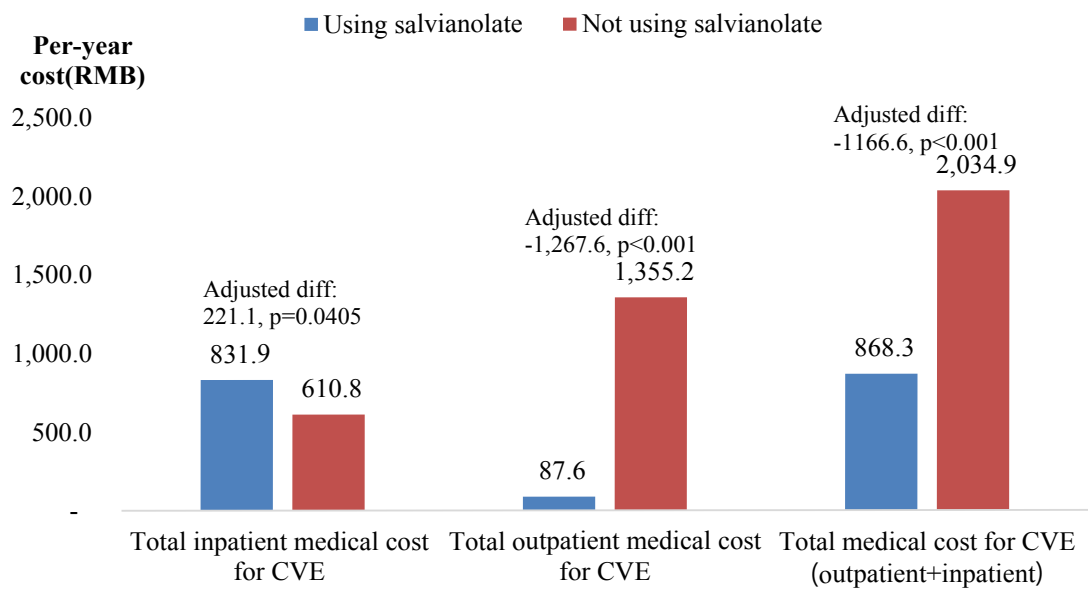

Abbreviations Diff: Difference; CNY: Renminbi, the Official Currency of China; MACE: Major Adverse Cardiovascular Event.

Figure 3 Total medical cost for MACE during 12 months follow up.

$$
\text { - using salvianolate not using salvianolate }
$$

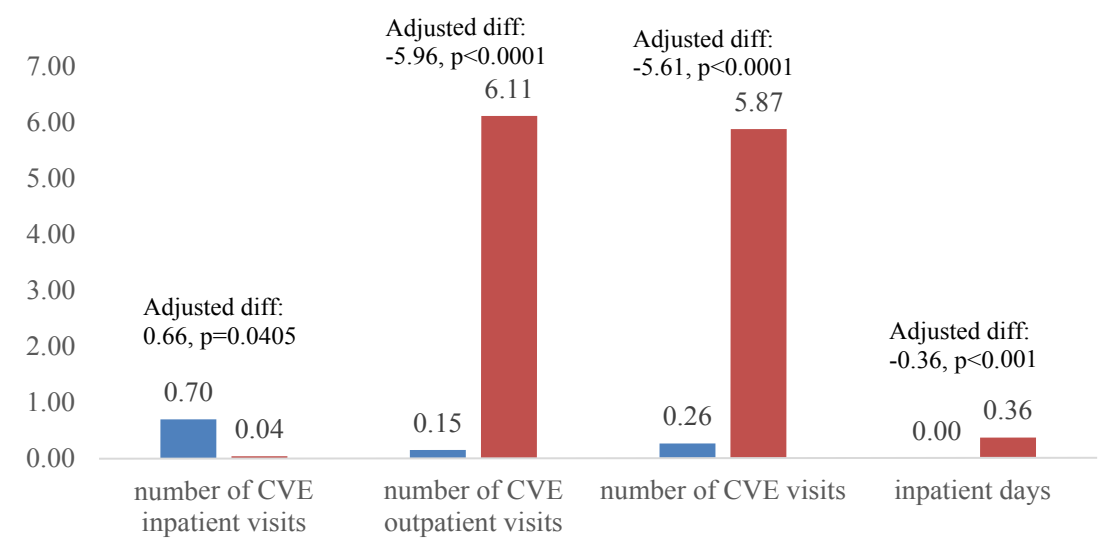

Abbreviations Diff: Difference; MACE: Major Adverse Cardiovascular Event.

Figure 4 Number of MACE during 12 months follow up.

\section{Discussions}

With the fast growing of aging population and urbanization, the incidence and mortality rate of coronary heart disease (CHD) would be continuing increasing, which would incurs more and more disease burdens in China. The number of coronary heart disease in Chinese adults aged 35-84 years is predicted to increase dramatically by $64 \%$ during the period of 2020-2029 [20]. The prevention and minimizing the cardiovascular events for patient already established coronary heart disease become more and more important for better quality of life and medial cost saving.

In our study, patient with coronary heart disease received Salvianolate injection showed significant total medical cost decreasing for all cause and Cardiovascular related events compared with patient not using salvianolate injection. During the follow-up period, the average number of cardiovascular events were 0.26 and 5.87 times for treatment and control group respectively ( $p<0.01$, [adjusted]). Compared with non-user, mean cardiovascular related per-year cost for salvianolate injection user was $29.58 \%$ lower (4069.21 vs. 5778.17 Chinese Yuan (CNY), and mean cardiovascular related per-year cost for salvianolate injection user was $57.33 \%$ lower (868.35 vs. 2,034.93 Chinese Yuan (CNY)) (Table 2).

Pengxin Dong, etcs retrospectively studied hospitalized patients with CHD from August 2011 to December 2015 by using electronic medical record database. The result showed that compared with conventional treatment of CHD, combination of salvianolate injection and conventional treatment with hospital stay significantly decreased by 2.9 days $(P<0.05)$ and total nitrates dosage significantly decreased by $172.4 \mathrm{mg}(P<0.05)[21]$. Our study indicated the consistent result with Dong's research.

Salvianolate injection is composed of salvianolic extraction from Danshen, Danshen is a very important component of Chinese 
Table 2 Cardiovascular-related resource use and total medical costs (CNY).

\begin{tabular}{|c|c|c|c|}
\hline & \multirow[t]{2}{*}{ Using Salvianolate ( $n=439)$} & \multirow{2}{*}{$\begin{array}{l}\text { Not Using Salvianolate } \\
(n=1848)\end{array}$} & \multirow{2}{*}{\begin{tabular}{|l|} 
Adjustec \\
P Value \\
\end{tabular}} \\
\hline & & & \\
\hline Missing value, NO. & 36 & 24 & \\
\hline Total inpatient medical costs, mean (SE), CNY & 2858.76(814.97) & 1468.25(64.88) & 0.593 \\
\hline Total outpatient medical costs, mean(SE), CNY & $74.20(11.36)$ & $7400.09(2071.36)$ & $<0.001$ \\
\hline Total medical costs, mean (SE), CNY & $4069.21(2117.28)$ & $5778.17(1032.64)$ & $<0.001$ \\
\hline Total inpatient medical cost for MACE, mean (SE), CNY & $831.93(20.07)$ & $610.83(9.85)$ & 0.842 \\
\hline Total outpatient medical cost for MACE, mean (SE), CNY & 87.64 (1.79) & 1355.22(11.92) & $<0.001$ \\
\hline $\begin{array}{l}\text { Total medical cost（outpatient and inpatient） for MACE, mean (SE), } \\
\text { CNY }\end{array}$ & $868.35(21.76)$ & 2034.93(27.12) & $<0.001$ \\
\hline
\end{tabular}

Abbreviation CNY: Renminbi, the Official Currency of China; SE: Standard Error of Mean; MACE: Major Adverse Cardiovascular Event; No.: Number

medicine and have been applicated in cerebrovascular disease treatment for many years. Besides Salvianolate injection, traditional Chinese medicine (TCM) shows great potential for general treatment for CHD. Comparing with western medicine, TCM functions through multiple paths and multiple target spots, so that it is always expected to improve patients' overall health status.

The real world pharmacoeconomics of TCM products is still at the very early development stage, and it needs further exploratory investigations. Our study analysed the total MACE rate and total cost difference from a real-world perspective to provide economic evidence to support optimal clinical treatment decisions for CHD patients.

A broadly recognized challenge with the analysis of real-world data is the need to correct for sample selection bias [22]. Generalized linear models and negative binomial regression adjusting for covariates, including demographics, baseline characteristics, i.e., previous drug usage, baseline patient clinical status, costs, and health care resource utilization, were used to improve baseline comparability between the two groups. Calculation of total medical costs and resource use could be underestimated.

\section{References}

1 WHO (2017) Key fact of cardiovascular disease.

2 Benjamin EJ, Blaha MJ, Chiuve SE, Cushman M, Das SR, et al. (2017) Heart disease and Stroke Statistics-2017 Update: A Report from the American Heart Association 135: e146-e603.

3 National Cardiovascular Center. China Cardiovascular Disease Report 2016 [M]. Beijing: China Encyclopedia Publishing House 2017.

4 China Health and Family Planning Commission. China Health and Family Planning Statistical Yearbook 2016 [M]. Beijing: China Union Medical University Press 2016.

5 Wong ND (2014) "Epidemiological studies of CHD and the evolution of preventive cardiology" Nature Reviews. Cardiology 11: 276-289.

6 Mendis, Shanthi, Puska, Pekka, Norrving, et al. (2011) global atlas on cardiovascular disease prevention and control. Geneva: World Health Organization in collaboration with the World Heart Federation and the World Stroke Organization. pp. 3-18.

7 Mehta PK, Wei J, Wenger NK (2015) "Ischemic heart disease in women: A focus on risk factors" Trends in Cardiovascular Medicine 25: $140-151$
The medical costs calculated in this study only include those happened within the study hospitals. Expense incurred in other hospital or pharmacies are not included, which may result in underestimation of the costs.

With the limitation of current database, we obtained data from two tier 3 hospitals in Shanghai and Guangzhou, both cities belong to Tier 1 cities. In future study, with more data availability, we would be able to further this study in small and middle size hospitals in broad geographic coverage in China to get more comprehensive view of the comparison result.

\section{Conclusion}

Among Chinese coronary heart disease and angina pectoris patients, those who used salvianolate injection had lower peryear cardiovascular event rate and spent less on cardiovascular events treatment.

\section{Funding Source}

The project is supported by the Foundation of Special topics of Shanghai Science and Technology Commission (No.15DZ1900300).

8 Charlson FJ, Moran AE, Freedman G, Norman RE, Stapelberg NJ, et al. (2013) "The contribution of major depression to the global burden of ischemic heart disease: a comparative risk assessment". BMC Medicine 11: 250.

9 Chinese guidelines for the prevention of cardiovascular diseases (2017) Chin J Cardiol 2018.

10 Wang Y, Xuan L (2005) Demonstration Achievements in Modernization of Traditional Chinese Medicine-Research and Development of Danshen Polyphenolate and Salvia Polyphenolate for Injection [J]. Chinese Journal of the Academy of Sciences 20: 377-380.

11 Qu J, Ren X, Hou R, PingDaia X, ChunZhao Y, et al. (2011) the protective effect of magnesium lithospermate $B$ against glucoseinduced intracellular oxidative damage [J]. Biochem Biophys Res Commun 411: 32-39.

12 Dingyou Li, Lina Xu, Xiaoguang Liu (1995) Effects of water-soluble components isolated from salvia miltiorrhiza on oxygenase free radical generation and lipid peroxidation. J Chin Pharm Sci 4: 107-112.

13 Hur KY, Kim SH, Choi MA, Williams DR, Lee YH, et al. (2010) Protective effects of magnesium lithospermate $B$ against diabetic atherosclerosis via Nrf2-ARENQO1 transcriptional pathway, Atherosclerosis, 211: 69-76. 
14 Meng C, Zhuo XQ, Xu GH, Liu JL (2014) Protection of salvianolate against atherosclerosis via regulating the inflammation in rats. J Huazhong Univ Sci Technol Med Sci. 34: 646-651.

15 Fei A, Cao Q, Chen S, Wang H, Wang F, et al. (2013) Salvianolate inhibits reactive oxygen species production in $\mathrm{H} 2 \mathrm{O} 2$-treated mouse cardiomyocytes in vitro via the TGF- $\beta$ pathway. Acta Pharmacol Sin 34: 496-500.

16 Han B, Zhang X, Zhang Q, Zhao G, Wei J, et al. (2011) Protective effects of salvianolate on micro vascular flow in a porcine model of myocardial ischaemia and reperfusion. Arch Cardiovasc Dis 104: 313-324.

17 Yang M, Zhuyu G, Fengqin X, Xiaofang W, Keyuan C, et al. (2006) Clinical study of Danshen polyphenolate in the treatment of coronary heart disease with angina pectoris (cardiovascular stasis syndrome) [J]. Journal of New Drugs and Clinical Pharmacy 17: 140-144.
18 Zhang D, Wu J, Liu S, Zhang X, Zhang B, et al. (2016) Salvianolate injection in the treatment of unstable angina pectoris: a systematic review and meta-analysis. Medicine 95: e5692.

19 Liu Y, Yan YY, Zhai SD (2016) Salvianolate for treatment of angina: an overview of systematic reviews. Chin J Clin Pharmacol 32: 560-562

20 Zhou M, Wang H, Zhu J, Chen W, Wang L, et al. (2016) Cause-specific mortality for 240 causes in China during 1990-2013: a systematic subnational analysis for the Global Burden of Disease Study 2013[J]. Lancet 387: 251-272.

21 Dong P, Hu H, Guan X, Shi L, Han S, et al. (2018) Cost-consequence analysis of salvianolate injection for the treatment of coronary heart disease. Chin Med 13: 28.

22 Garrison LP Neumann PJ, Erickson P, Marshall D, Mullins CD (2007) Using real-world data for coverage and payment decisions: the ISPOR Real-World Data Task Force report. Value Health 10: 326-335. 